\title{
Determination of the Interior Orientation Parameters of a Non-metric Digital Camera for Terrestrial Photogrammetric Applications*
}

\author{
${ }^{1}$ N. D. Tagoe and ${ }^{1}$ S. Mantey \\ ${ }^{1}$ University of Mines and Technology, Tarkwa, Ghana
}

Tagoe, N. D. and Mantey, S., (2019), "Determination of the Interior Orientation Parameters of a Non-metric Digital Camera for Terrestrial Photogrammetric Applications”, Ghana Mining Journal, Vol. 19, No. 2, pp. 1-9.

\begin{abstract}
High cost of metric photogrammetric cameras has given rise to the utilisation of non-metric digital cameras to generate photogrammetric products in traditional close range or terrestrial photogrammetric applications. For precision photogrammetric applications, the internal metric characteristics of the camera, customarily known as the Interior Orientation Parameters, need to be determined and analysed. The derivation of these parameters is usually achieved by implementing a bundle adjustment with self-calibration procedure. The stability of the Interior Orientation Parameters is an issue in terms of accuracy in digital cameras since they are not built with photogrammetric applications in mind. This study utilised two photogrammetric software (i.e. Photo Modeler and Australis) to calibrate a non-metric digital camera to determine its Interior Orientation Parameters. The camera parameters were obtained using the two software and the Root Mean Square Errors (RMSE) calculated. It was observed that Australis gave a RMSE of $\mathbf{0 . 2 4 3 5}$ and Photo Modeler gave 0.2335, implying that, the calibrated non-metric digital camera is suitable for high precision terrestrial photogrammetric projects.
\end{abstract}

Keywords: Camera Calibration, Interior Orientation Parameters, Non-Metric Digital Camera

\section{Introduction}

Interior orientation defines the internal geometries of a camera as it existed at the time of image capture. These parameters include: position of the principal point in the image plane (xo, yo), calibrated principal distance of the camera or the focal length of the lens and lens distortion parameters (Perez et al., 2011). In traditional aerial photogrammetry, a pre-calibrated metric camera attached to an aircraft is used for image acquisition. Non-metric digital cameras which are often not calibrated are also used in most terrestrial photogrammetric applications for image acquisition. Camera calibration is the process of determining the internal orientation parameters of a camera. These parameters include the principal point, the focal length of the lens and lens distortion. Unlike metric cameras that have been specifically designed for photogrammetric applications and have very stable interior orientation parameters and low lens distortion values, non-metric digital cameras have unstable interior orientation parameters and high lens distortion values (Fraser, 2006). A nonmetric camera can be turned into a precision instrument by determining its interior orientation parameters regularly, prior to and just after a project. A camera is considered calibrated when its true internal orientation parameters such as the principal distance or focal length of the lens, principal point and lens distortion parameters are known (Fraser, 2006). Lens distortion occurs when light rays passing through the lens are bent, thereby changing the directions and intersecting the image plane at positions deviant from the norm. It causes imaged positions to be displaced from where they ought to be. Though a number of lens distortion models are available (Hamid and Ahmad, 2014; Fryskowska et al., 2016; Salvi et al., 2002; Fraser and Al-Ajlouni, 2006; Läbe and Förstner, 2004; Fryer, 1996; Fraser, 2001; Bösemann et al., 1990; Fraser and Shortis, 1995; Kunii and Chikatsu, 2001; Shortis and Beyer, 1997; Peipe and Stephani, 2003; Wiley and Wong, 1995; D’Apuzzo and Maas, 1999), the commonly adopted methods are those of Tsai, (1986; 1987), Heikkila and Silven (1997), Zhang (2000) and Brown (1971). The Brown lens distortion model is widely used by photogrammetrist to model distortion characteristics of a camera lens (Brown, 1971). With the Brown model, the mathematical equations are typically of two components: the symmetric radial lens distortion and the decentering lens distortion. The symmetric radial lens distortion causes straight lines to appear as curved lines while the decentering distortion is the displacement of a point in the image caused by misalignment of the components of the lens. This study sought to determine the interior orientation parameters of a non-metric camera, namely the principal point coordinates, the focal length of the lens and lens distortion parameters.

\subsection{Cameras for Photogrammetric Project}

There are several types of cameras used for photogrammetric projects. The choice of camera is largely dependent on the objectives and the class of work to be produced (dependent on the accuracy to be achieved) whether first class, second class or third class. The duration and the cost involved may also influence the choice of camera. Cameras could be categorised as Red Green Blue cameras, 
Multispectral Cameras and Hyperspectral Cameras. Red Green Blue cameras only capture the primary colours or mostly all the colour part of pictures or photographs. They are not recommended for high precision work. Multispectral cameras usually have several lenses on board that could capture several pixels of overlapping photographs at different angles. Hyperspectral cameras use narrow, and usually contiguous spectral bands, involving possibly hundreds or thousands of spectra. Both multispectral and hyperspectral cameras are used for high precision works. Spectral cameras are cameras that could capture and analyse hidden features of images as well as internally broken or fractured images. They may include infrared or near infrared cameras. Some suggested cameras for photogrammetric projects are described briefly as follows.

\subsubsection{Rollei d7 Metric Camera}

The Rollei D7 metric camera (Fig. 1) is used for digital image acquisition. This 5-megapixel camera (2552 x 1920 pixel with approximately $3.5 \mu \mathrm{m}$ pixel spacing) provides some features of a metric camera such as a fixfocus lens ( $7 \mathrm{~mm}$ nominal focal length) and a rigid connection between lens and CCD sensor. The camera geometry can be assumed stable when capturing series of images (Peipe and Stephani, 2003).

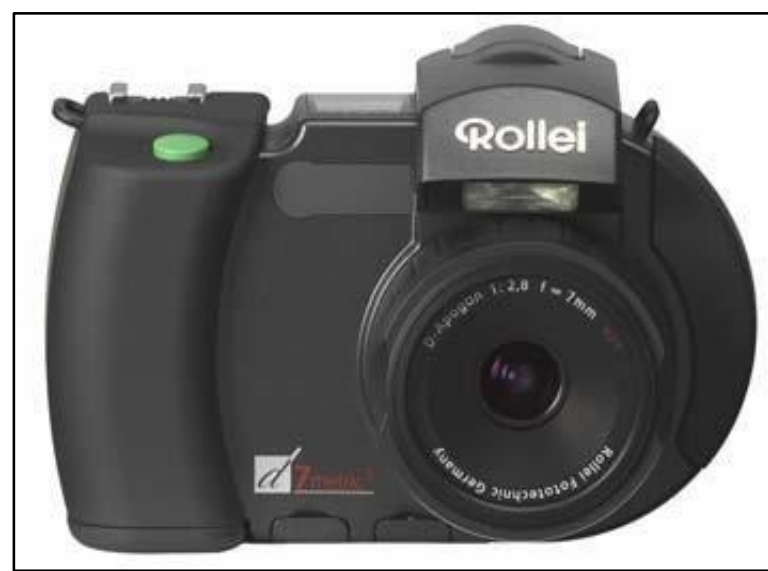

Fig. 1 Rollei d7 Metric Camera (Source: Peipe and Stephani, 2003)

\subsubsection{Nikon 3dc Metric Camera}

The Nikon 3dc Metric camera (Fig. 2) is a digital image acquisition camera. This 8-megapixel camera (1300 x 5740) pixel with approximately $2.5 \mu \mathrm{m}$ pixel spacing) provides some features of a metric camera such as a fix focus lens ( $5 \mathrm{~mm}$ nominal focal length) and a rigid connection between lens and CCD sensor as well as automatic shutter function after focusing. The camera geometry can also be assumed stable during capture of series of images (Peipe and Stephani, 2003).



Fig. 2 Nikon 3dc Metric Camera (Source: Peipe and Stephani, 2003)

\subsection{Camera Calibration}

Photogrammetric camera calibration is usually carried out together with the calculation of object coordinates within a self-calibrating bundle adjustment. The quality of the result depends mainly on the image configuration. If the network geometry is not adequate to self-calibration requirements, a prior knowledge of the camera parameters is needed for the object reconstruction. In photogrammetry, a series of commercially available programs exists to solve the calibration task (Peipe and Tecklenburg, 2006). This study is not intended to investigate or compare the accuracy of the final 3D coordinates, computing time or cost of the different software packages. Accurate calibration of cameras is especially crucial for applications that involve quantitative measurements such as dimensional measurements, depth from stereoscopy or motion from images (Weng et al., 1992). According to Brown (1971), one aspect of camera calibration is to determine the interior parameters of the camera. These parameters determine how the image coordinates of a point are derived, given the spatial position of the point with respect to the camera. The estimation of the geometrical relation between the camera and the scene, or between different cameras, is also an important aspect of calibration. The corresponding parameters that characterise such a geometrical relation is called external parameters. It is well known that cameras are not perfect and sustain a variety of aberrations. For geometrical measurements, the main concern is camera distortion, which relates to the position of image points in the image plane but not directly to the image quality. For example, the position of a point in a slightly blurred image can still be measured as the centre of the blurred point. However, if the image position of a point is not accurate, the results 
that depend on its image coordinates will be erroneous.

\subsection{Camera Parameters}

There are two main parameters used in the calibration of cameras. These include intrinsic and extrinsic parameters.

\subsubsection{Intrinsic and Extrinsic Parameters}

The intrinsic or internal parameters are mostly used for the correction of internal distortions of the camera lens (Hartley and Zisserman, 2000). They are: focal length of the lens, principal point (centre), pixel size and distortion coefficients. The extrinsic parameters are: rotation and translation parameters (Fig. 3). The extrinsic parameters define the location and orientation of the camera with respect to the external world.

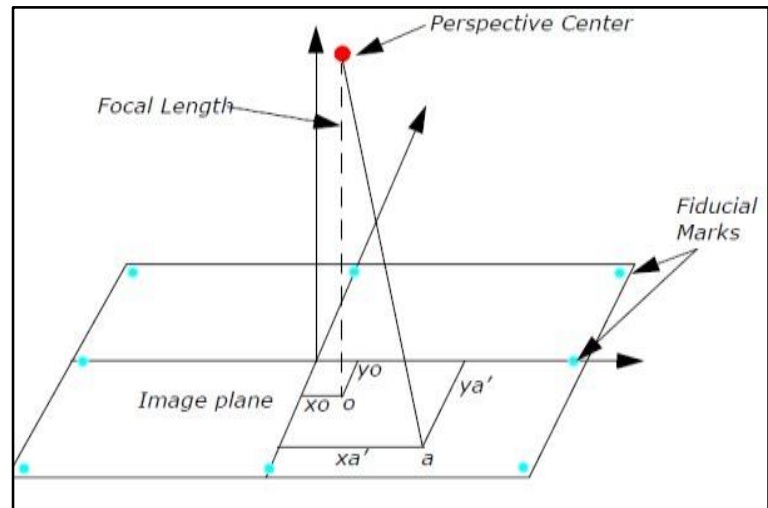

Fig. 3 Camera Parameters (Source: Hartley and Zisserman, 2000

\subsection{Principal Point and Focal Length}

The principal point is mathematically defined as the intersection in the image plane of the perpendicular line from the perspective centre. The focal length of the lens is the length from the principal point to the perspective centre.

\subsection{Lens Distortion}

According to Weng et al., (1992), geometrical distortion refers to the position of image points in the image plane. There are two major types of camera lens distortions. These are Radial lens distortion (Fig. 4) and Decentering lens distortion (Fig. 5).

\subsubsection{Radial Lens Distortion}

According to Barreto et al., (2003), radial lens distortion causes an inward or outward displacement of a given image point from its ideal location as shown in Fig. 4. This type of distortion is mainly caused by flawed radial curvature of the lens elements. A negative radial displacement of the image points is referred to as barrel distortion. It causes outer points to crowd increasingly together and the scale to decrease. A positive radial displacement is referred to as pincushion distortion. It causes outer points spread and the scale to increase. This type of distortion is strictly symmetric about the optical axis. Ideal image points are distorted along radial directions from the distortion centre (Ganapathy, 1984; Prescott and McLean, 1997). This distortion is caused by imperfect lens shape. Accurate radial lens distortion correction can be applied based on only approximate estimates of the aspect ratio and principal point (Lenz and Tsai 1987; Hartley and Zisserman, 2000).

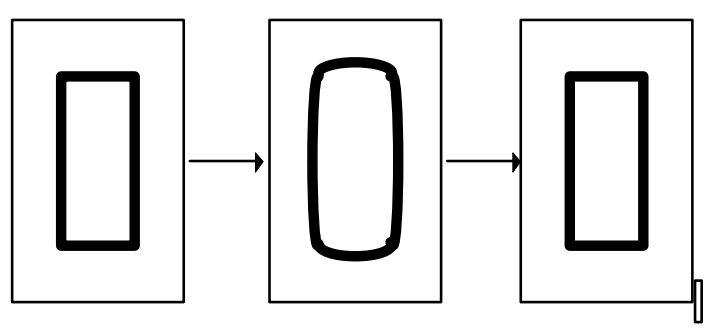

Fig. 4 Radial Distortion of Lens from Scene to Correction (Source: Hartley and Zisserman, 2000)

\subsubsection{Decentering Lens Distortion}

Decentering lens distortion is usually caused by improper lens assembly, that is, ideal image points are distorted in both radial and tangential directions. Actual optical systems are subject to various degrees of decentering, that is, the optical centres of lens elements are not strictly collinear. This defect introduces what is called decentering distortion (Faig, 1975; Fryer, 1996). Fig. 5 shows a perfectly centered lens and a decentered lens.

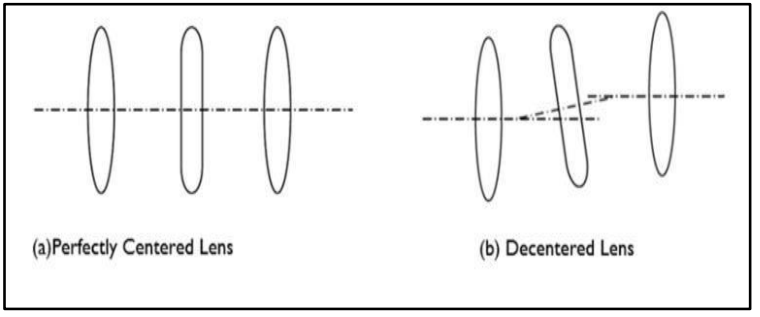

Fig. 5 Perfectly Centered Lens and Decentered Lens (Source: Hartley and Zisserman, 2000)

\subsection{Bundle Adjustment}

Bundle Adjustment describes a method of solving large minimisation problems on the basis of least squares (Brown, 1958; Barzilai and Borwein, 1988; Wu et al., 2011). This is the first known method for bundle adjustment and was used to minimise the 
projection error after determining point coordinates and camera positions from aerial images (Liu et al., 2012; Liu et al.,2008). Bundle adjustment was adapted and applied to close-range photogrammetry. Today it is one of the essential modules in virtually every Structure from Motion (SfM) pipeline. SfM aims to recover the position of the cameras as well as the $3 \mathrm{D}$ information of sparse points from a given set of images. The derived parameters are prone to inaccuracies caused by wrong correspondences, critical camera configurations (e.g. small baselines), measurement noise, and calibration errors. Furthermore, optimisation is carried out for certain problem subsets (either subset of images) (Agarwal et al., 2011) or subset of parameters (Moulon et al., 2013, Wilson, and Snavely, 2014). This leads to solutions that are optimal only for the corresponding subtasks instead of being optimal for the whole task. Bundle Adjustment aims to minimise these errors efficiently by performing a global optimisation process that considers all cameras and points. This optimisation process implies the formation and solving of equation systems, which becomes particularly computationally expensive for modern datasets involving hundreds of cameras. Therefore, bundle adjustment is often seen as (one of) the bottlenecks of corresponding reconstruction pipelines. Over the past few years, many approaches have been proposed that aim to optimise the efficiency of Bundle Adjustment, either on an algorithmic level or by usage of multi-core systems.

\section{Resources and Methods Used}

\subsection{Resources}

The resources used in this study are discussed in the subsequent sub headings.

\subsubsection{Non-Metric Digital Camera}

A 6.3-megapixel robust built quality Fujifilm FinePix camera (Fig. 6) was used in this study to take photographs of the calibration grid patterns at the appropriate angles.

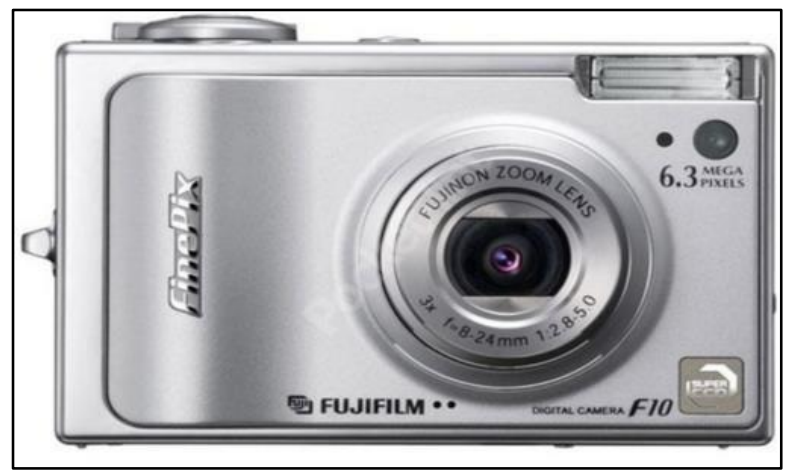

Fig. 6 Non-Metric Fujifilm FinePix Camera

\subsubsection{Calibration Grid Pattern}

A calibration grid pattern (Fig. 7) is a portable target field pattern, which is approximately $36 \times 36 \mathrm{~cm}$ in width comprising of $54 \times 3 \mathrm{~mm}$ diameter retroreflective targets. This was retrieved from a Photo Modeler's in-stored file directory.

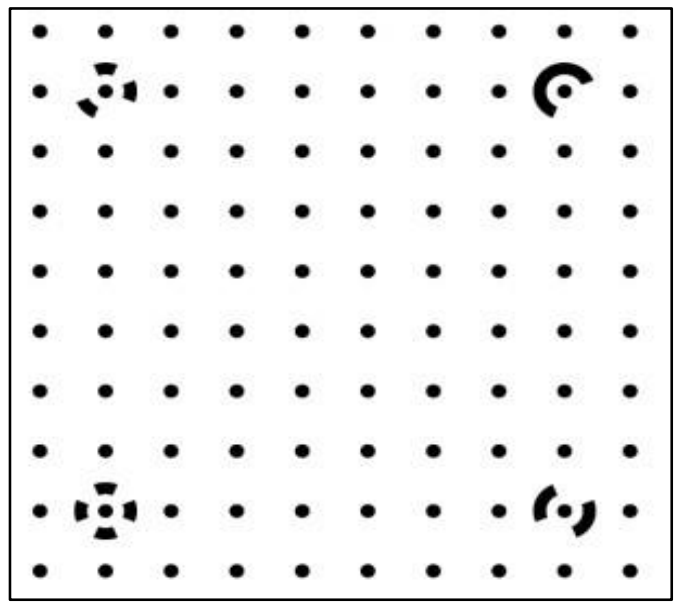

Fig. 7 Calibration Grid Pattern

\subsubsection{Photo Modeler Scanner}

Photo Modeler Scanner, a photogrammetric program developed by Earth Observing Systems was used in processing the images in this study. This relatively low-cost software is user friendly, has a broad range of applications and is designed for use by non-photogrammetric experts (Anon., 2018a).

\subsubsection{Australis Photogrammetric Software}

The Australis software is designed to perform highly automated 3D coordinate measurement and photogrammetric camera calibration from multi station digital networks. It is very intuitive and easy to operate as well as possessing a robust and reliable error detection through on-line data processing.

\subsubsection{GIMP}

GIMP is a free and open source image processing software used for image editing. It is good for image retouching and editing free form drawing, converting between different image formats and also for performing more specialised task (Anon., 2018b).

\subsection{Methods}

Fig. 8 is a flowchart showing the various methods employed in this study. 


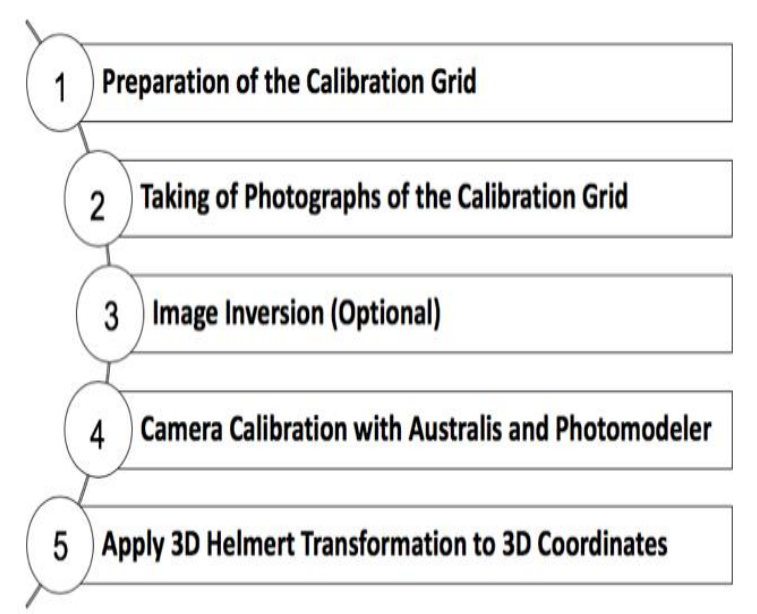

Fig. 8 Flowchart of Methods Used

\subsubsection{Preparation of the Calibration Grid}

The calibration grid (Fig. 7) obtained from the Photo Modeler scanners' in-stored file directory was displayed on a small paper size scale. It could however be scaled in any desired paper size and printed on a white paper background. It was printed on a larger paper to attain a higher accuracy.

\subsubsection{Taking of Photographs}

The printed grid was stationed on a flat surface where it could be visualised clearly. The calibration grid had four sides of which those four sides were chosen to be the four camera positions or stations (Fig. 9). The pictures were taken in three different orientations of the camera (Fig. 10). Thus, the camera was held in a landscape mode and then rotated 90 degrees to the left (portrait). It was brought back to landscape mode and then rotated 90 degrees to the right (portrait). This exercise was repeated for all the other remaining positions to obtain twelve photographs in all, with each side having three photographs taken at different orientations whilst the calibration grid remained stationary. Camera settings such as zoom, camera resolution and image quality were kept constant for all the photographs. The images were then transferred onto a computer.

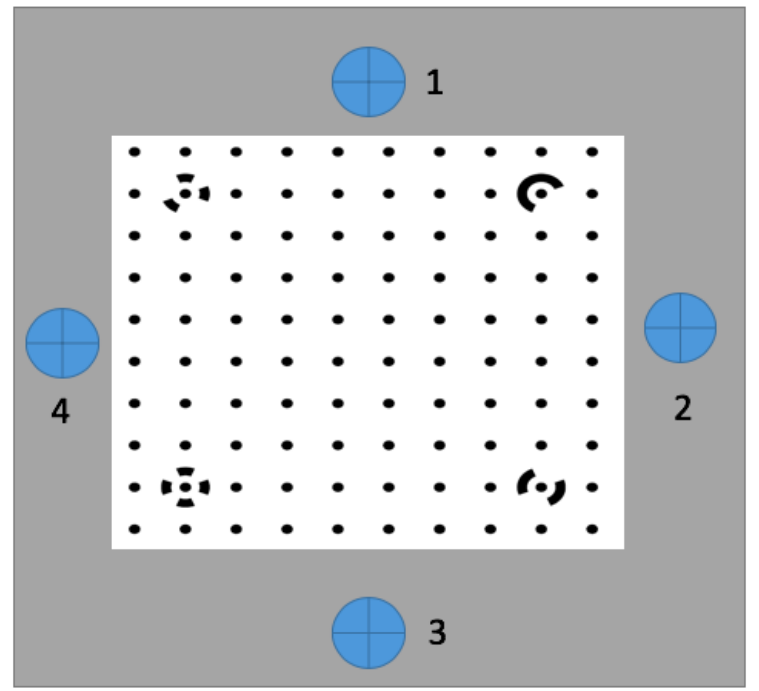

Fig. 9 Camera Positions with Respect to Calibration Grid



Fig. 10 Camera Orientations for Each Camera Position

\subsubsection{Image Inversion}

Photo Modeler scanner recognises black targets on white background while Australis recognises white targets on black background. Since the images obtained were generic to Photo Modeler scanner, there was the need to invert the images to obtained their negatives or the inverse for them to have worked appropriately in Australis. This was done using the GIMP image processing software where the images were loaded for further pre-processing (Fig. 11).

\section{Camera Calibration in Photo Modeler}

The calibration process was done by importing the twelve set of images with black target fields into the Photo Modeler scanner software and processing them. The processing interface of the Photo Modeler software is shown in Fig. 12. 


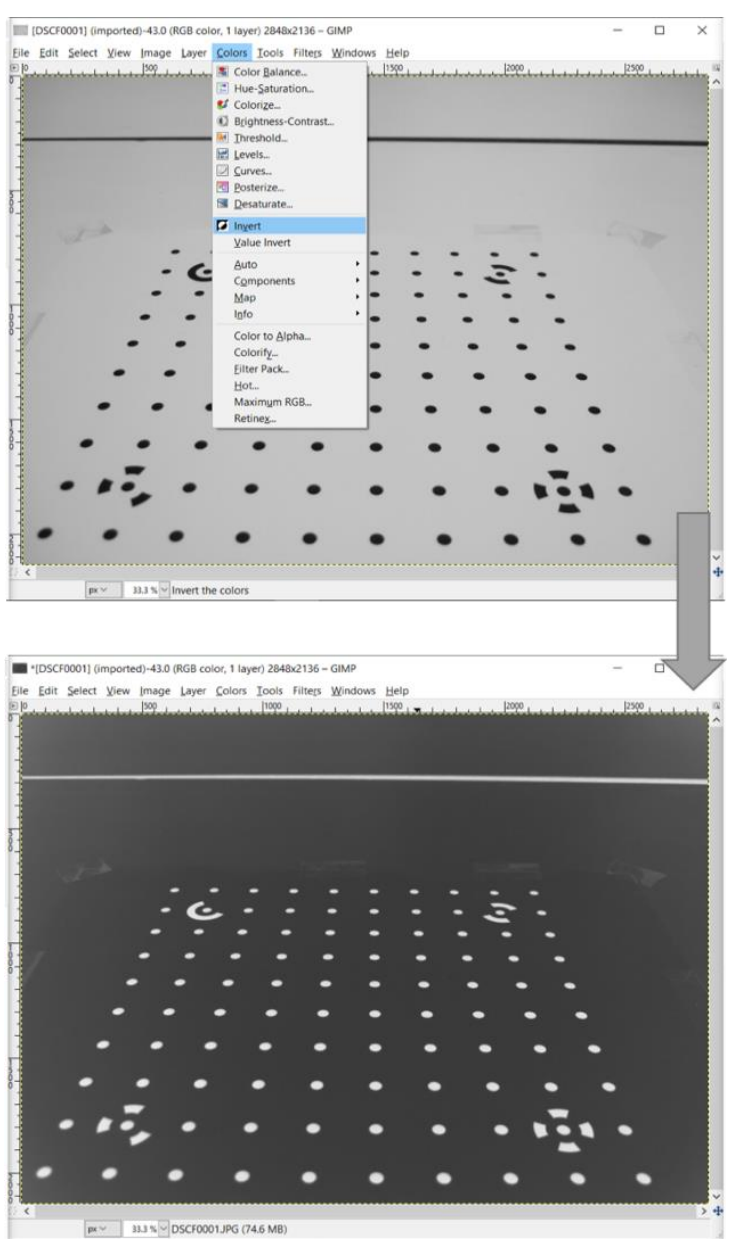

Fig. 11 Image Inversion with GIMP Software

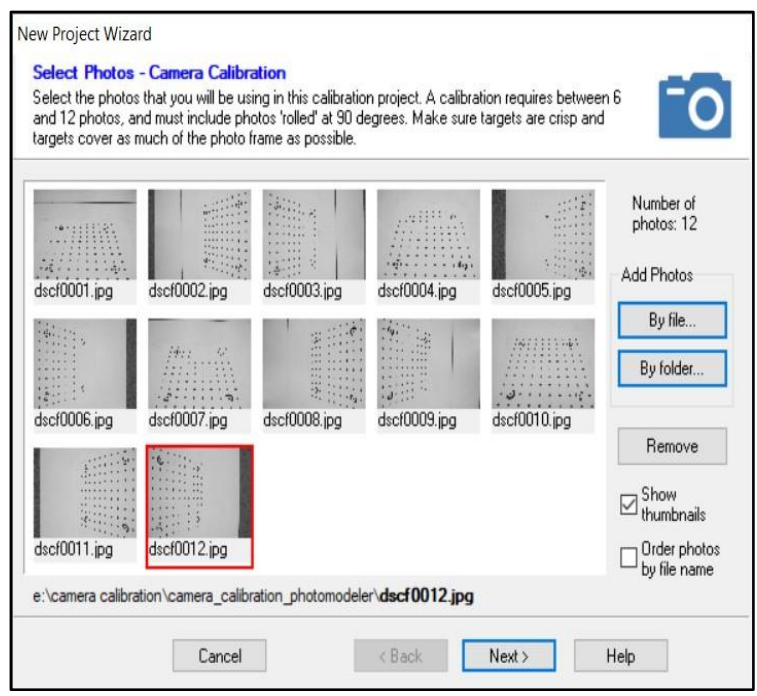

Fig. 12 Image Processing Interface in Photo Modeler

\section{Image Processing and Calibration in Australis}

Australis software on the computer desktop was launched and then a new project was created. The project units were defined in millimeters and saved. The information about the camera (Fig. 6) used for this study was added to the camera database after which the pre-processed images of the calibration grid with white target fields were added to the project. The calibration process was done by selecting all the images in the project and measuring all measurable points (Figs. 12 and 13) on the inversed calibration grid one after the other to determine their positions.

\begin{tabular}{|c|c|c|c|c|c|c|}
\hline \multicolumn{4}{|c|}{ Camera DataBase } & & & $x$ \\
\hline \multicolumn{7}{|c|}{$\begin{array}{l}\text { Calibration } \\
\text { Time }\end{array}$} \\
\hline \multicolumn{3}{|c|}{ Camera Id FuifilmF10 } & (0) & & $\begin{array}{l}\text { ensor Size } \\
\text { [pixels] }\end{array}$ & $\begin{array}{l}\text { Pixel Size } \\
\text { [mm] }\end{array}$ \\
\hline \multicolumn{2}{|c|}{$\begin{array}{l}\text { Type / } \\
\text { Serial \# }\end{array}$} & & & $\mathrm{H}$ & 2848 & 0.00263 \\
\hline \multicolumn{2}{|c|}{$\begin{array}{r}\text { Lens Type / } \\
\text { Serial \# }\end{array}$} & & & $v$ & 2136 & 0.00263 \\
\hline \multicolumn{2}{|r|}{ Current Value } & Sigma & Fix & \multicolumn{3}{|c|}{ Sigmas Option } \\
\hline [c] & 7.860241 & 1000 & $\Gamma$ & \multicolumn{3}{|c|}{ Fix All } \\
\hline \multirow{2}{*}{$\begin{array}{l}\text { (Xp] } \\
\text { YYp] }\end{array}$} & 0.020058 & 1000 & $\Gamma$ & \multicolumn{3}{|c|}{ Free All } \\
\hline & 0.043200 & 1000 & $\Gamma$ & \multicolumn{3}{|c|}{ Fix $K 1 \cdot B 2$} \\
\hline \multirow{2}{*}{ [K1] } & $1.1732 \mathrm{e}-003$ & 1000 & $\Gamma$ & \multirow{2}{*}{\multicolumn{3}{|c|}{ Fix K2-B2 }} \\
\hline & $1.0428 \mathrm{e}-004$ & 1000 & $\Gamma$ & & & \\
\hline (K3) & $-3.5752 e-006$ & 1000 & $\Gamma$ & \multirow{2}{*}{\multicolumn{3}{|c|}{ Fiducials }} \\
\hline (P1) & $\mid-7.6081 \mathrm{e}-005$ & 1000 & $\Gamma$ & & & \\
\hline (P2) & $\mid-2.5611 \mathrm{e}-004$ & 1000 & $\Gamma$ & \multirow{2}{*}{\multicolumn{2}{|c|}{$\begin{array}{c}\text { Save changes } \\
\text { to this } \\
\text { camera }\end{array}$}} & \\
\hline [B1] & $3.7115 \mathrm{e}-026$ & $1 \mathrm{e}-016$ & $\sqrt{\checkmark}$ & & & Ok \\
\hline (B2) & $\mid 5.7545 \mathrm{e}-027$ & $1 \mathrm{e}-016$ & $\sqrt{\checkmark}$ & & ave & Cancel \\
\hline
\end{tabular}

Fig. 13 Camera Parameters in Australis

\section{Bundle Adjustments and Distortion Parameters}

The images were processed by bundle adjustment based on collinearity equations (Equations 1 and 2) which has an input for lens distortion. The condition for collinearity is that the object point, the image point and the camera position also known as the perspective position all lie in a straight line as expressed in Equations 1 and 2 (Abdel-Aziz et al., 1971).

$x_{i}-x_{o}+\Delta x=c \frac{m_{11}\left(X_{j}-X_{\boldsymbol{o}}\right)+m_{12}\left(Y_{j}-Y_{\boldsymbol{o}}\right)+m_{13}\left(Z_{j}-Z_{\boldsymbol{o}}\right)}{m_{31}\left(X_{j}-X_{\boldsymbol{o}}\right)+m_{32}\left(Y_{j}-Y_{\boldsymbol{o}}\right)+m_{33}\left(Z_{j}-Z_{\boldsymbol{o}}\right)}$

$y_{i}-y_{o}+\Delta y=c \frac{m_{21}\left(X_{j}-X_{\boldsymbol{o}}\right)+m_{22}\left(Y_{j}-Y_{\boldsymbol{o}}\right)+m_{23}\left(Z_{j}-Z_{\boldsymbol{o}}\right)}{m_{31}\left(X_{j}-X_{\boldsymbol{o}}\right)+m_{32}\left(Y_{j}-Y_{\boldsymbol{o}}\right)+m_{33}\left(Z_{j}-Z_{\boldsymbol{o}}\right)}$

where:

$\mathbf{x}_{\mathbf{i}}, \mathbf{y}_{\mathbf{i}}$ are measured image coordinates;

$\mathbf{X}_{\mathbf{j}}, \mathbf{Y}_{\mathbf{j}}, \mathbf{Z}_{\mathbf{j}}$ are object space coordinates of the measured points;

$\mathbf{X}_{\mathbf{0}}, \mathbf{Y}_{\mathbf{0}}, \mathbf{Z}_{\mathbf{0}}$ are object space coordinates of the perspective centre of the camera (position of the camera); 
$\mathbf{m}_{11}$ to $\mathbf{m}_{33}$ are the individual elements of the orthogonal rotation matrix representing the threeangle omega, phi and kappa;

$\mathbf{c}$ is the focal length of lens;

$\mathrm{X}_{0}, \mathrm{y}_{0}$ are principal points of coordinates;

$\Delta x, \Delta y$ are lens distortion parameters.

The bundle adjustment resulted in three dimensional coordinates of the points on the calibration grid. These three-dimensional coordinates of the grid were compared with when they were in their idle positions and after they had been calculated. This was done by applying a 3D Helmert Transformation or the 3D Similarity Transformation /rigid-body transformation. The seven-parameter $3 \mathrm{D}$ coordinate transformation was used because the coordinates of the grid were not generated with respect to any datum thus, they were the rectangular coordinates. The seven-parameter 3D coordinate transformation is expressed in Equation 3:

$$
\mathrm{X}_{\mathrm{T}}=\mathrm{C}+\mu \mathrm{RX}
$$

where:

$\mathrm{X}_{\mathrm{T}}$ is the transformed matrix

$\mathrm{X}$ is the initial vector

$\mathrm{C}$ is the translation vector containing the three translation along the coordinate axes

$\mu$ is the scale factor

$\mathrm{R}$ is the rotation matrix consisting of three axes

\section{Results and Discussion}

\subsection{Results}

The camera parameters obtained after calibrating with the Photo Modeler scanner and Australis are tabulated in Table 1 and Table 2 respectively.

Table 1 Camera Parameters after Calibration in Photo Modeler

\begin{tabular}{|l|c|}
\hline \multicolumn{1}{|c|}{ Parameter } & Value \\
\hline $\mathrm{C}$ (Focal length) & 8.0058 \\
\hline $\mathrm{X}_{\mathrm{P}}$ (Principal point X) & 3.9197 \\
\hline $\mathrm{Y}_{\mathrm{P}}$ (Principal point $\left.\mathrm{Y}\right)$ & 2.9125 \\
\hline $\mathrm{K}_{1}$ (Radial distortion 1) & $1.205_{\mathrm{e}}-003$ \\
\hline $\mathrm{K}_{2}$ (Radial distortion 2) & $9.81 \mathrm{e}_{\mathrm{e}}-006$ \\
\hline $\mathrm{P}_{1}$ (Decentering distortion 1$)$ & $-1.561_{\mathrm{e}}-004$ \\
\hline $\mathrm{P}_{2}$ (Decentering distortion 2$)$ & $2.092_{\mathrm{e}}-005$ \\
\hline \multicolumn{1}{|c|}{ RMSE } & 0.2435 \\
\hline
\end{tabular}

Table 2 Camera Parameters after Calibration in Australis

\begin{tabular}{|l|c|}
\hline \multicolumn{1}{|c|}{ Parameter } & Value \\
\hline $\mathrm{C}$ (Focal length) & 7.9579 \\
\hline $\mathrm{X}_{\mathrm{P}}$ (Principal point $\left.\mathrm{X}\right)$ & 0.0246 \\
\hline $\mathrm{Y}_{\mathrm{P}}$ (Principal point $\left.\mathrm{Y}\right)$ & 0.0263 \\
\hline $\mathrm{K}_{1}$ (Radial distortion 1$)$ & $1.1909_{\mathrm{e}}-003$ \\
\hline $\mathrm{K}_{2}$ (Radial distortion 2$)$ & $4.5640_{\mathrm{e}}-006$ \\
\hline $\mathrm{P}_{1}$ (Decentering distortion 1$)$ & $-1.2582_{\mathrm{e}}-004$ \\
\hline $\mathrm{P}_{2}$ (Decentering distortion 2$)$ & $1.4702_{\mathrm{e}}-005$ \\
\hline \multicolumn{1}{|c|}{ RMSE } & 0.2335 \\
\hline
\end{tabular}

\subsection{Discussion}

The differences between individual parameters from the results obtained from both Photo Modeler scanner and Australis software were not significant, however they were not compared due to the different coordinate systems in which each of the software were developed and depicted in Figs. 1 and 2. It was observed that Australis gave a Root Mean Square Error (RMSE) value of $\mathbf{0 . 2 3 3 5}$ while Photo Modeler gave a Root Mean Square Error (RMSE) value of 0.2435. This depicts that Australis and Photo Modeler could be used to calibrate non-metric cameras and determine their interior orientation parameters for high accuracy projects.

\section{Conclusions and Recommendation}

\subsection{Conclusions}

In conclusion, a non-metric digital camera (Fujifilm FinePix f10) was calibrated using Photo Modeler scanner and Australis photogrammetric software and their interior orientation parameters derived makes it suitable for carrying out precision terrestrial photogrammetric projects. The overall root mean squares of the two software after calibrating the camera were also determined. Australis gave a Root Mean Square Error (RMSE) of $\mathbf{0 . 2 3 3 5}$ as while that of Photo Modeler gave a Root Mean Square Error (RMSE) of 0.2435.

\subsection{Recommendation}

It is recommended that non-metric digital cameras for carrying out photogrammetric projects and most optical instruments with variable internal geometries used for various surveys should be calibrated from time to time, at least once in a year in order to render them suitable for precision photogrammetric projects. 


\section{References}

Abdel-Aziz, Y. I. and Karara, M. M. (1971), “Direct linear transformation into object space coordinates in Close-Range Photogrammetry," Proceedings of the ASPRS Symposium on Close Range Photogrammetry, Urbana, IL, USA, 2829 January 1971; pp. 1-18.

Agarwal, S., Furukawa, Y., Snavely, N., Simon, I., Curless, B.,Seitz, S. M. and Szeliski, R., (2011), "Building Rome in a Day", Communications of the Association for Computing Machinery, Vol. 54, Issue 10, pp. 105-112.

Anon., (2018a), "Photo Modeler 2018", http://www.photomodeler.com/. Accessed:April 10, 2018, pp. 1-5.

Anon., (2018b), “About GIMP”, http://www.gimp.org/about/introduction.html Accessed: April 17, 2018 pp. 50-58.

Barreto J. P., Martin F., and Horaud, R. (2003), "Visual Servoing/Tracking Using Central Catadioptric Images", Experimental Robotics VIII., B. Siciliano and P.Dario (Ed).Springer Verlag, triangulation", Technical Report 43, RCA Data reduction, 23pp.

Barzilai, J. and Borwein, J., (1988), “Two-point step size gradient methods", IMA Journal of Numerical Analysis, Vol. 8 No. 1, pp. 141-148.

Bösemann, W., Godding, R., and Riechmann, W., (1990), "Photogrammetric investigation of CCD cameras. In Close Range Photogrammetry Meets Machine Vision, Gruen and Baltsavias (Edition), SPIE, Vol. 1395, pp. 119-126.

Brown, D. C., (1958). "A solution to the general problem of multiple station analytical stereo triangulation", Technical Report 43, RCA Data reduction, $23 \mathrm{pp}$.

Brown, D. C., (1971), "Close-range camera calibration”, Photogrammetric Engineering, Vol. 37, Issue 8, pp. 855-866.

D'Apuzzo, N. and Maas, H. G., (1999), "On the suitability of digital camcorders for virtual reality image data capture", Proceedings of SPIE Videometrics VI, El-Hakim and Gruen (Edition), Vol. 3461, pp. 259-267.

Faig, W. (1975), "Calibration of Close-Range Photogrammetric Systems: Mathematical formulation," Photogrammetric Engineering and Remote Sensing, Vol. 41, No. 12, pp. 1479-1486.

Fraser, C.S., and S. Al-Ajlouni, (2006), "Zoomdependent camera calibration in close-range photogrammetry", Photogrammetric Engineering and Remote Sensing, pp. 1017-1026.

Fraser, C. S. and Shortis, M., (1995), "Metric exploitation of still video imagery", The Photogrammetric Record, Vol. 15, No. 85, pp. 107-122.

Fraser, C.S., (2001), "Photogrammetric camera component calibration. A review of analytical techniques. In 'Calibration and Orientation of
Cameras in Computer Vision', Springer Series in Information Sciences, Vol. 34, pp. 95-121.

Fryer, J. G. (1996), “Close Range Photogrammetry and Machine Vision", Whittles Publishing, Scotland, Atkinson, pp. 156-179.

Fryskowska, A., Kedzierski, M., Grochala, A., and Braula, A. (2016), "Calibration of low cost rgb nir uav cameras", In 23rd International Society for Photogrammetry and Remote Sensing Congress, Prague, Czech Republic, pp. 817-821.

Ganapathy, S. (1984), "Decomposition of transformation matrices for robot vision," in Proceedings of IEEE International Conference on Robotics and Automation. (Atlanta), pp. 130139.

Hamid, N. F. A., and Ahmad, A. (2014). Calibration of high-resolution digital camera based on different photogrammetric methods. IOP Conference Series: Earth and Environmental Science, Vol. 18, No. 1, pp. 1-6.

Hartley, R. I and Zisserman, A. (2000), "Multiple View Geometry in Computer Vision", Cambridge University Press, Second Edition, 646pp.

Heikkilä, J. and Silven, O., (1997), “A four-step camera calibration procedure with implicit image correction", Proceedings of IEEE Computer Society Conference on Computer Vision and Pattern Recognition, pp. 1106-1112.

Kunii, Y. and Chikatsu, H., (2001), "On the application of 3 million consumer digital camera to digital photogrammetry", Proceedings of SPIE Videometrics VII, Vol. 4309, pp. 278-287.

Läbe, T. and Förstner, W., (2004), "Geometric stability of low-cost digital consumer cameras", International Archives of Photogrammetry, Remote Sensing and Spatial Information Sciences, Vol. 35(5), pp 528-535.

Lenz, R. K. and Tsai R. Y. (1987), "Techniques for calibration of the scale factor and image centre for high accuracy 3D machine vision metrology," in Proceedings of the IEEE International Conference on Robotics and Automation (Raleigh, NC), pp. 68-75.

Liu, S., Sun, J. and Dang, J., (2008), "A linear resection-intersection bundle adjustment method", Information Technology Journal, Vol. 7, Issue 1, pp. 220-223.

Liu, X., Gao, W. and Hu, Z.-Y. (2012), "Hybrid parallel bundle adjustment for $3 \mathrm{~d}$ scene reconstruction with massive points", Journal of Computer Science and Technology, Vol. 27, Issue 6, pp. 1269-1280.

Moulon, P., Monasse, P. and Marlet, R., (2013). "Global fusion of relative motions for robust, accurate and scalable structure from motion". In Computer Vision and Pattern Recognition, IEEE, pp. 3248-3255.

Peipe, J. and Tecklenburg, W., (2006), "Photogrammetric Camera Calibration Software 
- A Comparison', Institute for Photogrammetry and Geoinformatics, Vol. 3, No. 22, 75pp.

Peipe, J. and Stephani, M., (2003), "Performance evaluation of a 5-megapixel digital metric camera for use in architectural photogrammetry", International Archives of Photogrammetry, Remote Sensing and Spatial Information Sciences, Vol. 34 (5/W12), pp. 259261.

Perez, M., and Aguera, F. C. F. (2011), "Digital camera calibration using images taken from an unmanned aerial vehicle", The International Archives of the Photogrammetry, Remote Sensing and Spatial Information Sciences, Vol. 38, No. 1, pp. 1-5.

Prescott, B. and McLean, G. F. (1997), "Line-based correction of radial lens distortion", Graphical Models Image Process, Vol. 59, Issue 1, pp. 3947.

Salvi, J., Armanguè, X. and Batlle, J., (2002), “A comparative review of camera calibration methods with accuracy evaluation", Pattern Recognition, Vol. 35, pp. 1617-1635.

Shortis, M., and Beyer, H.A., (1997), "Calibration stability of the Kodak DCS420 and 460 cameras", Proceedings of SPIE Videometrics V, Vol. 3174, 12pp.

Tsai, R.Y., (1986), “An efficient and accurate camera calibration technique for 3D machine vision", Proceedings of IEEE on Computer Vision, pp. 364 - 374.

Tsai, R.Y., (1987), “A versatile camera calibration technique for high-accuracy $3 \mathrm{D}$ machine vision metrology using off-the-shelf TV cameras and lenses", IEEE International Journal of Robotics and Automation, Vol. 3, No. 4, pp. 323-344.

Weng, J., Cohen, P. and Herniou, M. (1992), "Camera Calibration with Distortion Models and Accuracy Evaluation," IEEE Close-Range Photogrammetry, (Urbana, IL), pp. 1-5.

Wiley, A. G. and Wong., K. W., (1995), “Geometric calibration of zoom lenses for computer vision metrology", Photogrammetric Engineering and Remote Sensing, Vol. 61, No. 1, pp. 69-74.

Wilson, K. and Snavely, N., (2014), "Robust global translations with $1 \mathrm{dsfm",} \mathrm{In:} \mathrm{European}$ Conference on Computer Vision, Springer, pp. 61-75.

Wu, C., Agarwal, S., Curless, B. and Seitz, S. M., (2011), Multicore bundle adjustment. In: Computer Vision and Pattern Recognition, IEEE Computer Society, pp. 3057-3064.

Zhang, Z., (2000), "A flexible new technique for camera calibration", IEEE Transaction on Pattern Analysis and Machine Intelligence, Vol. 22, No. 11, pp. 1330-1334.

\section{Authors}

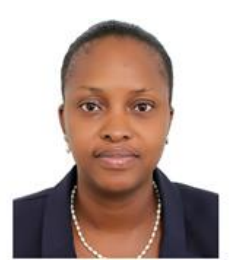

Naa Dedei Tagoe is a Senior Lecturer at the Department of Geomatic Engineering of the University of Mines and Technology (UMaT), Tarkwa, Ghana. She was awarded BSc. degree in Geodetic Engineering at KNUST, Ghana. She obtained her MSc. degree in Photogrammetry and Geoinformatics from Stuttgart University of Applied Sciences, Germany. She also holds $\mathrm{PhD}$ in Geomatics from University of Cape Town. She is a Member of IFUW and a Licensed Surveyor (No. 374). Her research interests include Close Range Photogrammetry, 3D Modelling of Cultural Heritage Sites, Remote Sensing, UAVs and WebGIS Applications.



Saviour Mantey is a Senior Lecturer and a Licensed Surveyor (No. 371). at the Department of Geomatic Engineering of the University of Mines and Technology (UMaT), Tarkwa, Ghana. He holds a BSc degree in Geodetic Engineering from KNUST, Ghana. He obtained his Master of Philosophy degree and Doctor of Philosophy from University of Cambridge and University of Mines and Technology respectively. He is a Licensed Drone Pilot an Instructor (GCAA/RPAS No. 007). His research interest includes application of Remote Sensing and GIS in Health and Environmental Analysis, UAVs and WebGIS applications. 